\title{
A Feasibility Study of a Prototype PET Insert Device to Convert a General-Purpose Animal PET Scanner to Higher Resolution
}

\author{
Heyu $\mathrm{Wu}^{1,2}$, Debashish Pal ${ }^{3}$, Joseph A. O’Sullivan ${ }^{4}$, and Yuan-Chuan Tai ${ }^{1}$ \\ ${ }^{I}$ Mallinckrodt Institute of Radiology, Washington University, St. Louis, Missouri; ${ }^{2}$ Institute of Modern Physics, Chinese Academy of \\ Science, Lanzhou, China; ${ }^{3}$ Department of Biomedical Engineering, Washington University, St. Louis, Missouri; and \\ ${ }^{4}$ Department of Electrical and System Engineering, Washington University, St. Louis, Missouri
}

We developed a prototype system to evaluate the feasibility of using a PET insert device to achieve higher resolution from a general-purpose animal PET scanner. Methods: The system consists of a high-resolution PET detector, a computer-controlled rotation stage, and a custom mounting plate. The detector consists of a cerium-doped lutetium oxyorthosilicate array $(12 \times$ 12 crystals, $0.8 \times 1.66 \times 3.75 \mathrm{~mm}^{3}$ each) directly coupled to a position-sensitive photomultiplier tube (PS-PMT). The detector signals were fed into the scanner electronics to establish coincidences between the 2 systems. The detector was mounted to a rotation stage that is attached to the scanner via the custom mounting plate after removing the transmission source holder. The rotation stage was concentric with the center of the scanner. The angular offset of the insert detector was calibrated via optimizing point-source images. In all imaging experiments, coincidence data were collected from 9 angles to provide $180^{\circ}$ sampling. A ${ }^{22} \mathrm{Na}$ point source was imaged at different offsets from the center to characterize the in-plane resolution of the insert system. $A{ }^{68} \mathrm{Ge}$ point source was stepped across the axial field of view to measure the sensitivity of the system. A 23.2-g mouse was injected with $38.5 \mathrm{MBq}$ of ${ }^{18} \mathrm{~F}$-fluoride and imaged at $3 \mathrm{~h}$ after injection for $2 \mathrm{~h}$. Results: The transverse image resolution of the PET insert device ranges from 1.1- to 1.4-mm full width at half maximum (FWHM) without correction for the point-source dimension. This corresponds to approximately $33 \%$ improvement over the resolution of the original scanner (1.7- to $1.8-\mathrm{mm}$ FWHM) in 2 of the 3 directions. The sensitivity of the device is $0.064 \%$ at the center of the field, 46 -fold lower than the sensitivity of an existing animal PET scanner. The mouse bone scan had improved image resolution using the PET insert device over that of the existing animal PET scanner alone. Conclusion: We have demonstrated the feasibility of using a highresolution insert device in an existing PET scanner to provide high-resolution PET. A PET insert device with more detector modules will improve sensitivity and may become an alternative to special-purpose PET systems for high-resolution PET.

\footnotetext{
Received Jun. 12, 2007; revision accepted Sep. 29, 2007.

For correspondence or reprints contact: Yuan-Chuan Tai, PhD, Washington University School of Medicine, Campus Box 8225, $510 \mathrm{~S}$. Kingshighway Blvd., St. Louis, MO 63110.

E-mail: taiy@wustl.edu

COPYRIGHT @ 2008 by the Society of Nuclear Medicine, Inc.
}

Key Words: small-animal PET; high-resolution PET; PET insert; animal imaging

J Nucl Med 2008; 49:79-87

DOI: 10.2967/jnumed.107.044149

$\mathbf{T}$ he development of high-resolution PET systems has been an active research area since the mid-1990s (1-11). The unique capability of quantitative imaging using highresolution PET scanners has stimulated scientists' use of small-animal imaging techniques for biomedical research. This development has triggered a new field of molecular imaging, and scientists are now studying human disease formation and progression and developing new interventions more effectively using animal models $(12,13)$.

Although many research groups focus their efforts on development of new detector materials and detector design using conventional PET system geometry, we have proposed a novel geometry for PET-namely, the "virtualpinhole PET geometry" (14). A PET system based on this geometry can use 2 types of detectors with different intrinsic spatial resolutions. High-resolution detectors are positioned near the object, whereas low-resolution detectors are positioned far away from the object. Coincidence lines of response between these 2 types of detectors form a fanbeam geometry with the high-resolution detector elements at the vertices of the fans. If the high- and lowresolution detectors are rotated around the object to collect coincidence events from different angles, high-resolution tomographic images of the radioactivity distribution within the object can be reconstructed despite the presence of low-resolution detectors in the system. This is, indeed, very similar to a SPECT system that uses a low-resolution camera and a pinhole collimator to achieve high-resolution imaging within a reduced imaging field of view.

The simplest implementation of a virtual-pinhole PET system is through the use of 2 sets of PET detectors and a coincidence-detection processor. The high-resolution detector can be made of fine scintillation crystals and a light 
detector with high spatial resolving capability, such as a position-sensitive photomultiplier tube (PS-PMT). The low-resolution detectors can be made of coarse scintillation crystals and 4 single-channel PMTs to provide large solidangle coverage and still maintain a relatively low cost. The 2 sets of detectors rotate around an object to enable tomographic imaging. This approach is conceptually simple and inexpensive to implement. However, the rotation requirement makes the system less ideal for dynamic imaging applications that are often important to the users of animal PET technique. An alternative approach is to use all detectors in a general-purpose animal PET scanner as the "low-resolution" detectors. By incorporating one or more high-resolution detectors into the system and establishing coincidence detection between these 2 types of detectors, we would be able to implement the virtual-pinhole PET geometry and achieve the desirable higher resolution through the use of an existing animal PET scanner.

In this work, we describe our first prototype system that was built to prove the feasibility of using an "insert" device to achieve higher-resolution images through an existing animal PET scanner using the virtual-pinhole PET geometry. The detector and system design, as well as software development, are described in detail. Image resolution and sensitivity of the system were measured. A mouse bone scan was acquired to demonstrate the potential improvement in image resolution for this kind of device.

\section{MATERIALS AND METHODS}

\section{Description of microPET F-220 Scanner}

The existing animal PET scanner used was the microPET F-220 scanner (Siemens Medical Solutions, Inc.). The system consists of 168 detector modules arranged in 4 rings of $256 \mathrm{~mm}$ in diameter. Each detector consists of a cerium-doped lutetium oxyorthosilicate $\left(\mathrm{Lu}_{2}\left(\mathrm{SiO}_{4}\right) \mathrm{O}: \mathrm{Ce}\right.$ [LSO] $)$ crystal array, an optical fiber bundle, and a position-sensitive photomultiplier tube (PS-PMT). Each LSO array consists of 12 by 12 crystals, each measuring $1.51 \times$ $1.51 \times 10.00 \mathrm{~mm}^{3}$. The crystal pitch is $1.6 \mathrm{~mm}$ in both transverse and axial directions. Each detector module is read out by an independent channel of electronics in the base cabinet. A coincidence processor compares singles events from all detectors and transfers qualified coincidence events to the host computer to be stored in a list-mode file (15).

\section{Description of Insert Device}

The prototype insert device comprises one detector module, a computer-controlled rotation stage, a mounting bracket, and a host personal computer that controls the motion of the rotation stage and communicates with the microPET console for data acquisition.

The detector module consists of an LSO array directly coupled to a PS-PMT (R5900-C12; Hamamatsu Photonics). The LSO array contains 12 by 24 crystals, each measuring $0.8 \times 0.8 \times 3.75$ $\mathrm{mm}^{3}$ with a pitch of $0.86 \mathrm{~mm}$. Because the microPET F-220 scanner uses LSO arrays of 12 by 12 crystals, the electronics and firmware of the scanner were designed to handle 144 crystals per detector module. For the scanner to handle our detector signals correctly, we treated our LSO array as a $12 \times 12$ array by grouping 2 crystals into one with its dimension $=0.8$ (transverse $) \times 1.66$ (axial) $\times 3.75$ (radial) $\mathrm{mm}^{3}$. The PS-PMT and multiplexing scheme used in our detector are identical to those used in the F-220 system, which enable us to read out our detector signals using the existing system's electronics directly. By connecting the insert detector to an electronics channel associated with a detector near the top of the gantry, coincidence events between the insert detector and all detectors in the lower portion of the gantry can be detected through the coincidence processor in the scanner. Therefore, we disabled detector 83 in head 1 of the scanner (corresponding to the detector near the top of the gantry in the fourth ring) and used the electronics for this channel to process signals from our detector (Fig. 1A). Events detected by all other detectors in the fourth ring are discarded by our custom sorting program to mimic a situation in which a full-ring or half-ring insert system has multiple detectors and requires multiple detectors in the fourth rings to be disabled. As a result, the disabled detector(s) in the fourth ring does not cause missing data in sinograms because the scanner was treated as if it has only 3 full rings of detectors in coincidence with our insert device.

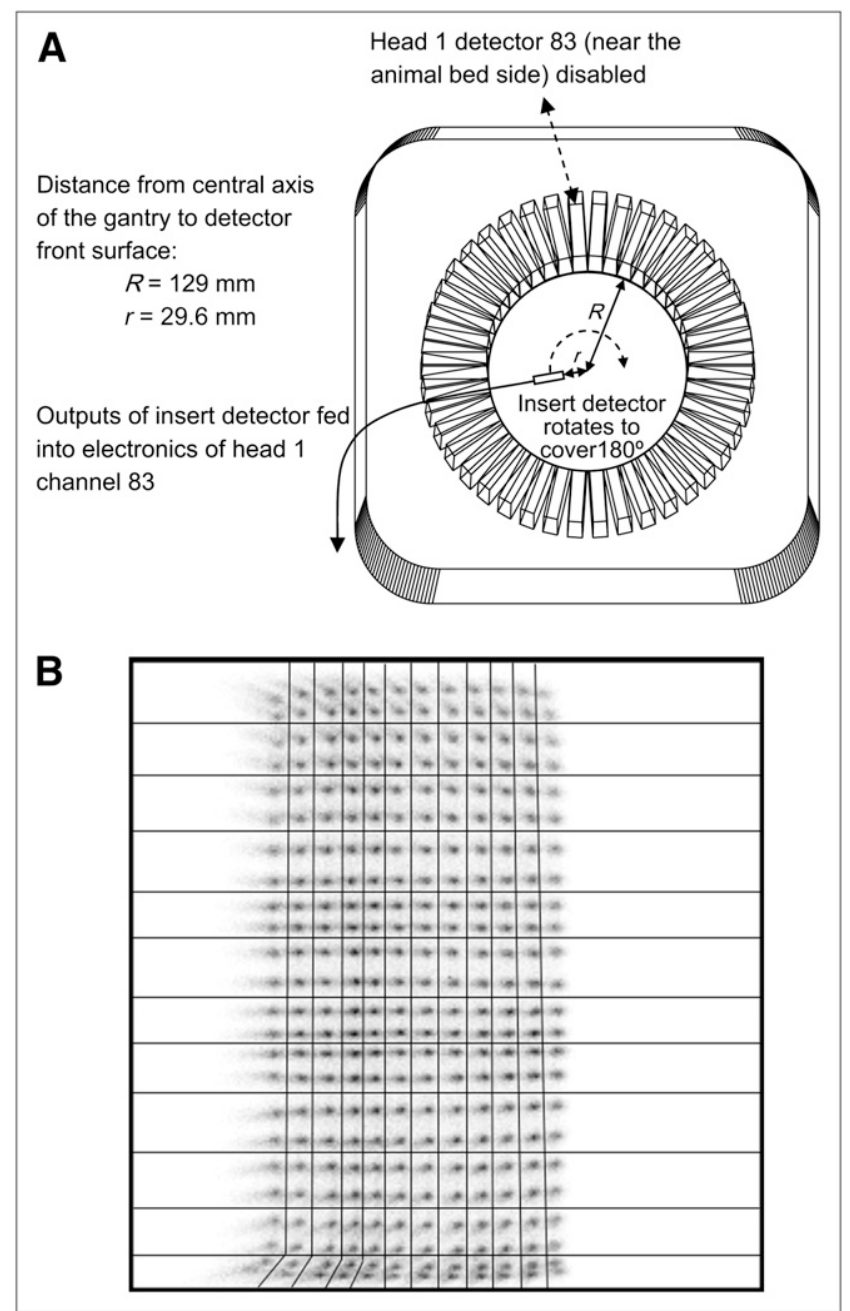

FIGURE 1. (A) Illustration of microPET F-220 gantry with an insert detector inside the animal port (base cabinet with electronics is not shown). (B) Flood image of high-resolution detector in the insert device with crystal lookup table overlaid on the image. 
To set up our detector, we first acquired a flood image of our detector using a ${ }^{68} \mathrm{Ge}$ source and manually created a lookup table for the 144 artificial crystals, as shown in Figure 1B. We then identified the photopeak location for individual artificial crystals and loaded this information to the system memory. From the system's perspective there are still 168 detector modules, and so it is not affected by the connection of an external detector to the system. However, any coincidence event that involves detector 83 in head 1 is actually coincidence between the insert device and the original microPET scanner. This type of coincidence event is referred to as an insert-scanner event, which provides high-resolution images using the virtual-pinhole PET geometry. Coincidence events between all other channels correspond to scanner-scanner coincidence - that is, the events normally measured by the micro PET F-220 scanner.

For the single-detector insert device to acquire tomographic images, our high-resolution detector must be rotated within the gantry to collect insert-scanner coincidence events at different angles. We attached the detector module on a custom mounting plate, which was itself mounted to a computer-controlled rotation stage on a custom-built bracket. This bracket and assembly can be attached to the back of the scanner at the same position the transmission source holder is normally found. The center of the rotation stage is concentric to the scanner gantry. The insert detector was located $29.6 \mathrm{~mm}$ from the center of rotation. The axial position of the insert detector was chosen so that the center of the insert detector is aligned with the center of the 3 active detector rings in the microPET F-220 system. Figure 2 shows the back and front views of the microPET F-220 system after the insert device is mounted in the gantry.

The host computer of the insert system controls the data acquisition of the microPET F-220 scanner and the motion of the rotation stage via a custom program based on LabView (National Instrument). This program communicates with a Javabased program residing in the console computer of the microPET F-220 scanner following standard Transmission Control Protocol and the Internet Protocol (TCP/IP protocol). The PET insert host computer can issue commands to the microPET F-220 console to backup its current setup files, download the setup files of the insert system to the scanner, as well as start list-mode data acquisition for an arbitrary amount of time. The same LabView-based program controls the rotation stage through a motion controller (PCI-7344; National Instrument) housed in the host computer. For a typical imaging experiment, the host computer first rotates the detector to a predefined location and then issues a command to the microPET console to acquire data for a predefined amount of time. This step-and-shoot motion repeats 9 times to provide $180^{\circ}$ sampling, instead of 18 times to provide $360^{\circ}$ sampling, because the insert detector cannot be in coincidence with detectors near the top of the gantry. This is because coincidences between detectors in the same bucket are discarded by the coincidence processor. Because our detector was connected to a channel near the top of the gantry, we rotated our detector within the upper half of the gantry opening to collect coincidence events with detectors in the lower portion of the gantry. The acquisition time at each angle is decay-corrected for the half-life of the radionuclide used in the experiment. When the experiment is completed, a user can use the same LabView-based program to restore the setup files of the microPET scanner so that the scanner can revert to its original state. Through this software package, the scanner can be switched to the PET insert mode or restored to its original functionality in $<10$ min. The initial setup of the PET insert system to create a crystal lookup table and to identify the photo peak of individual crystals needs to be done only once.

Once the list-mode data are acquired, coincidence events are processed by a custom sorting program to separate the events into 2 types (insert-scanner and scanner-scanner) and store them in 2 separate sinograms. We have adopted and implemented a 2-dimensional filtered-backprojection algorithm for the fourthgeneration $\mathrm{CT}$, which uses fanbeam geometry similar to the PET insert system (16). We also implemented a maximum-likelihood expectation maximization reconstruction algorithm that models the geometry of the insert system.

\section{Alignment and Calibration Procedures}

The mounting bracket of the insert device was designed and carefully machined to align the center of the rotation stage to the central axis of the microPET scanner gantry. When a detector is mounted on the rotation stage, however, its angular and axial positions relative to the existing detectors in the scanner are unknown. This information is required for the image reconstruction program and has to be carefully calibrated to obtain the best image resolution. Similar to the artifact in a SPECT system when its center of rotation is off, a point source may appear as a doughnut shape $\left(360^{\circ}\right.$ sampling $)$ or a tuning fork $\left(180^{\circ}\right.$ sampling $)$ in the reconstructed image if the parameter in the reconstruction program does not agree with the angular offset of the insert detector. This angular offset parameter was calibrated by putting a few point sources in the imaging field of view and the coincidence data acquired. The images were reconstructed with an initial estimate of the angular offset. The angular offset parameter was subsequently increased or decreased in the reconstruction program and the outer diameters of the tuning-fork-shaped point sources measured from the reconstructed images, as shown in Figure 3A. The diameter of the point source increases linearly as the estimated angular offset deviates from its true value in both
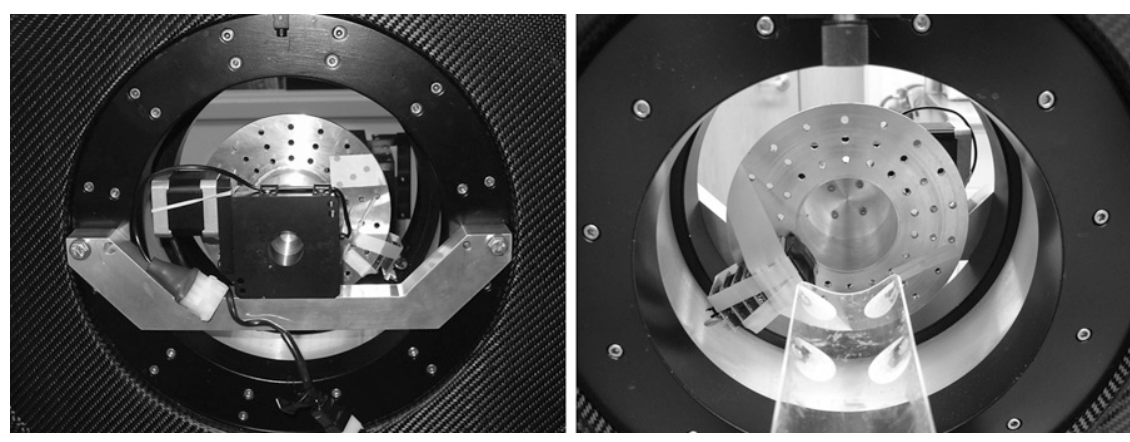

FIGURE 2. Prototype PET insert device can be mounted to microPET F-220 system after transmission source holder is removed from back of scanner (left). This prototype system consists of a single detector module and a rotation stage (right). Imaging field of view of PET insert device is $4 \mathrm{~cm}$ (transverse) by $2-3$ cm (axial; see sensitivity section). 


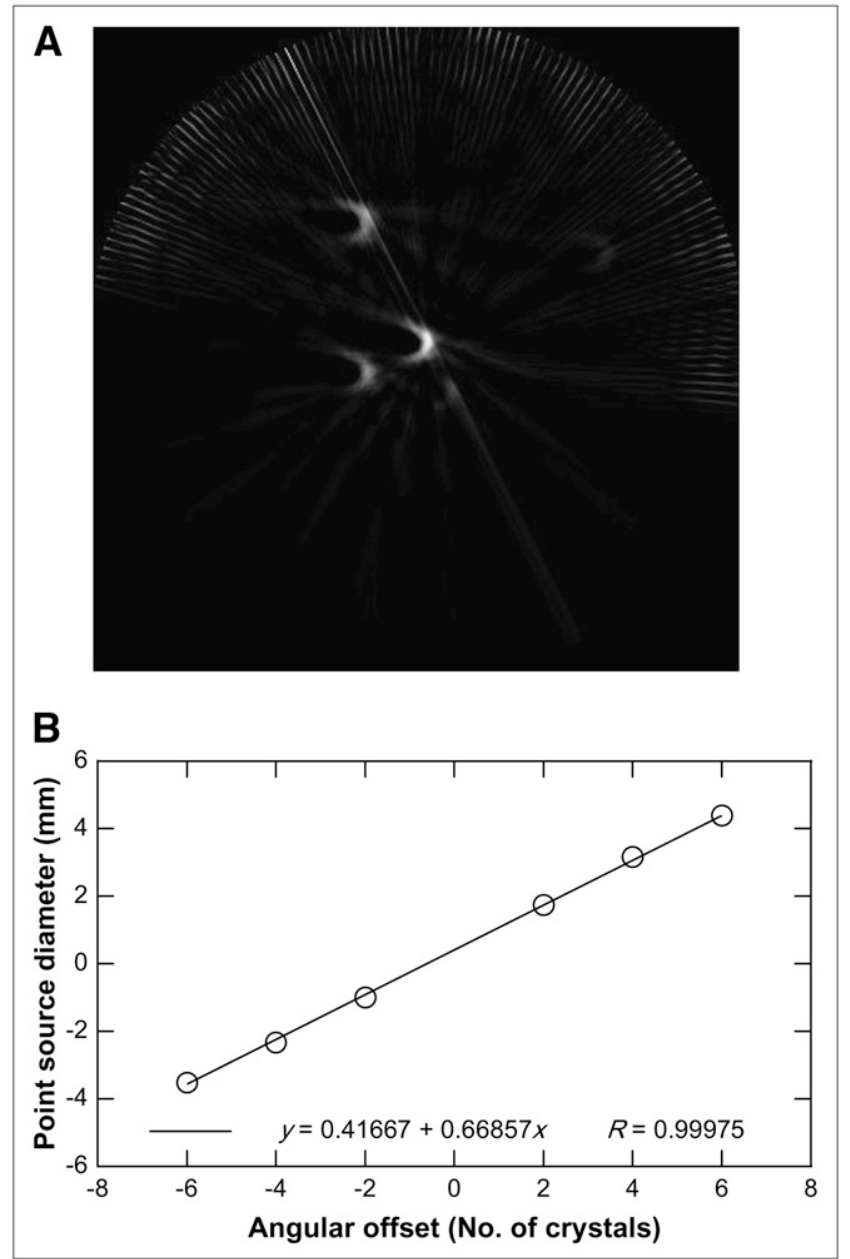

FIGURE 3. Calibration for alignment of insert detector with respect to scanner detectors. (A) Reconstructed image of point sources when center-of-rotation error is not corrected. (B) Fit the measured point-source diameter (diameter of half circle in A) as a function of angular offset between insert system and scanner by a straight line; zero-crossing point corresponds to correct angular offset that provides the highest image resolution to PET insert system. In this case, calibrated angular offset is $-0.297^{\circ}$, which equals -0.623 times the angle spanned by a single crystal in scanner detector.

positive and negative angles. If we consider the diameter to be a positive (negative) value when the angular deviation is along the positive (negative) direction, we could plot the size of the doughnut against the angular offset and fit the results with a straight line, as shown in Figure 3B. The angular offset that provides the highest reconstructed image resolution corresponds to the zero-crossing point of the fitted line. Through this calibration procedure, the insert system was set up after being mounted to the microPET F-220 scanner.

\section{Resolution Measurement}

$\mathrm{A}^{22} \mathrm{Na}$ point source $(0.5-\mathrm{mm}$ nominal diameter) embedded in a 25-mm diameter acrylic disk (North American Scientific) was used for all resolution measurements. The choice of ${ }^{22} \mathrm{Na}$ source was because its emission energy $\left(E_{\text {average }} \beta+=215 \mathrm{keV}\right)$ is similar to that of the most widely used positron-emitting isotope ${ }^{18} \mathrm{~F}\left(\mathrm{E}_{\text {average }} \beta+=250 \mathrm{keV}\right)$; yet the long half-life of ${ }^{22} \mathrm{Na}$ made it much easier to perform the resolution measurement. The source was first positioned at the center of the field of view in the PET insert system. The scanner was set to acquire coincidence events using an energy window of 250-750 keV and a coincidence timing window of $6 \mathrm{~ns}$. Data were collected for $72 \mathrm{~s}$ from each of the 9 angles that provide $180^{\circ}$ sampling and sorted into fanbeam sinograms using a single-slice rebinning algorithm (17). The measurements were repeated after repositioning the source at 2 , $4,6,8,10,12,14$, and $16 \mathrm{~mm}$ above the center of the field of view. The source was subsequently moved to the opposite half of the field of view $(2,4,6,8$, and $10 \mathrm{~mm}$ below the center of field of view), and the resolution measurements were repeated. This second set of measurements was necessary because the insert detector rotates $180^{\circ}$, not $360^{\circ}$. Therefore, the resolution of this particular PET insert system is asymmetric and depends on whether the source location is within the half ring spanned by the insert detector's trajectory or outside of the half ring. In these experiments, the upper half of the field of view is within the half ring spanned by the detector trajectory, whereas the lower half of the field of view is outside of the half ring.

Images were reconstructed by a fanbeam filtered backprojection algorithm that specifically models the geometry of our insert system (16). A ramp filter was applied with a cutoff frequency equal to the Nyquist frequency. Tangential and radial resolutions were estimated from the horizontal and vertical profiles through the pixel with maximum intensity in the image of each pointsource measurement. The full width at half maximum (FWHM) and full width at tenth maximum (FWTM) were measured from the extracted profiles by linear interpolation between the 2 nearest pixels bracketing the half values and one-tenth values, respectively. Results were plotted and compared with those previously reported for the microPET F-220 scanner using the same ${ }^{22} \mathrm{Na}$ point source (15). Resolution results reported were not corrected for source dimension, positron range, or acolinearity of positron annihilation.

\section{Sensitivity Measurement}

The sensitivity of the PET insert system was measured using a 2.11-MBq $(57 \mu \mathrm{Ci}){ }^{68} \mathrm{Ge}$ point source (1-mm nominal diameter) encapsulated in a stainless steel sleeve. The scanner was set to acquire coincidence events using an energy window of 250-750 $\mathrm{keV}$ and a coincidence-timing window of $6 \mathrm{~ns}$. The point source was stepped along the central axis of the field of view in multiple steps of $2 \mathrm{~mm}$ for a total of $3.2 \mathrm{~cm}( \pm 1.6 \mathrm{~cm}$ from the center of the field of view of the PET insert system). Data were collected for $60 \mathrm{~s}$ at each source location. Coincidences between the insert device and 3 rings of detectors in the microPET scanner (insertscanner coincidence events) were sorted into fanbeam sinograms. The total number of events in the insert-scanner sinogram was normalized to the total number of disintegrations in the source (corrected for the branching ratio of 0.891 for ${ }^{68} \mathrm{Ge}$ and approximately 5\% attenuation from the stainless steel sleeve) and then plotted as a function of source location to represent the system sensitivity profile along the central axis in the PET insert system.

\section{Imaging Experiment}

A 23.2-g mouse was injected with $38.5 \mathrm{MBq}$ of ${ }^{18} \mathrm{~F}$-fluoride and sacrificed at $3 \mathrm{~h}$ after injection. The mouse was positioned with its head centered in the image field of view and scanned from 9 angles that provide $180^{\circ}$ sampling. Acquisition time at the initial angles was approximately $9 \mathrm{~min}$, whereas the time for the subsequent angles was extended to compensate for radioactive 


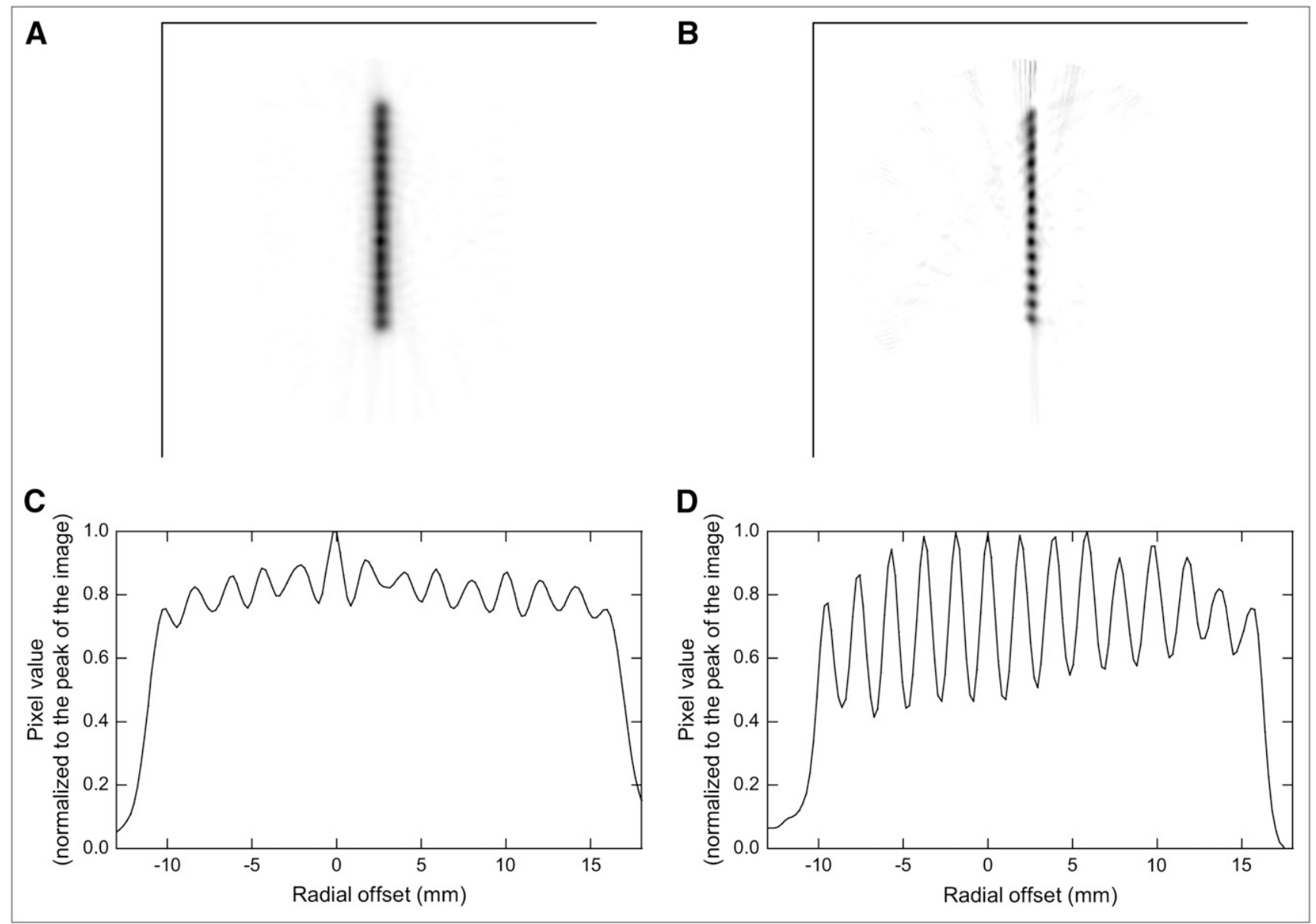

FIGURE 4. Composite image of point source measured at different locations (lowest point in image was located at $10 \mathrm{~mm}$ below center of field of view of the system). (A) Image from coincidences recorded by detectors in microPET F-220 scanner. (B) Image from coincidences between PET insert detector and detectors in scanner. (C and D) Line profiles through point sources in A and B, respectively.

decay of ${ }^{18} \mathrm{~F}$ in the mouse, with a total acquisition time of $120 \mathrm{~min}$. Insert-scanner coincidence events for all 9 angles were sorted into fanbeam sinograms and reconstructed by a 3-dimensional maximum-likelihood expectation maximization reconstruction algorithm that specifically models the PET insert geometry. The scanner-scanner coincidence events (from a single angle in $9 \mathrm{~min}$ ) were sorted into parallel-beam sinograms using Fourier rebinning (18) and reconstructed by an ordered-subset expectation maximization algorithm that is one of the standard reconstruction algorithms provided by the microPET F-220 system.

\section{RESULTS}

\section{Image Resolution of Insert System}

Figure 4 shows the composite image of the reconstructed images of the point source measured at different locations. Figure $4 \mathrm{~A}$ shows the image measured by the microPET F-220 scanner - that is, reconstructed using scanner-scanner coincidences. Figure 4B shows the image measured by the PET insert system-that is, reconstructed using insertscanner coincidences. The PET insert device has significantly higher image resolution than the F-220 scanner and can clearly separate point sources that are $2 \mathrm{~mm}$ apart near the central region of the imaging field of view, as can be seen in Figures 4C and 4D, which show line profiles through the point sources in Figures $4 \mathrm{~A}$ and $4 \mathrm{~B}$, respectively. As the point source moves up in the imaging field of view - that is, close to the PET insert device - the parallax error increases and the radial resolution starts to degrade. This can be better seen in Figure 5, where the tangential and radial resolutions of the PET insert device are compared with those of the microPET F-220 scanner previously reported (15). Note that the microPET F-220 scanner is circularly symmetric; thus, its image resolution of the upper half of the field of view should be identical to that of the lower half of its field of view. The resolution at negative radial offset locations replicates previously published data measured at positive radial offset locations. Figure 5A shows that the FWHM of the PET insert device is significantly better than the microPET F-220 system near the center of the field of view (1.1- to 1.2-mm FWHM vs. 1.7to $1.8-\mathrm{mm}$ FWHM). However, the resolution of this PET insert system is asymmetric for the upper half and the lower half of the imaging field of view. In the upper half (positive radial offset), the radial resolution degrades quickly and 

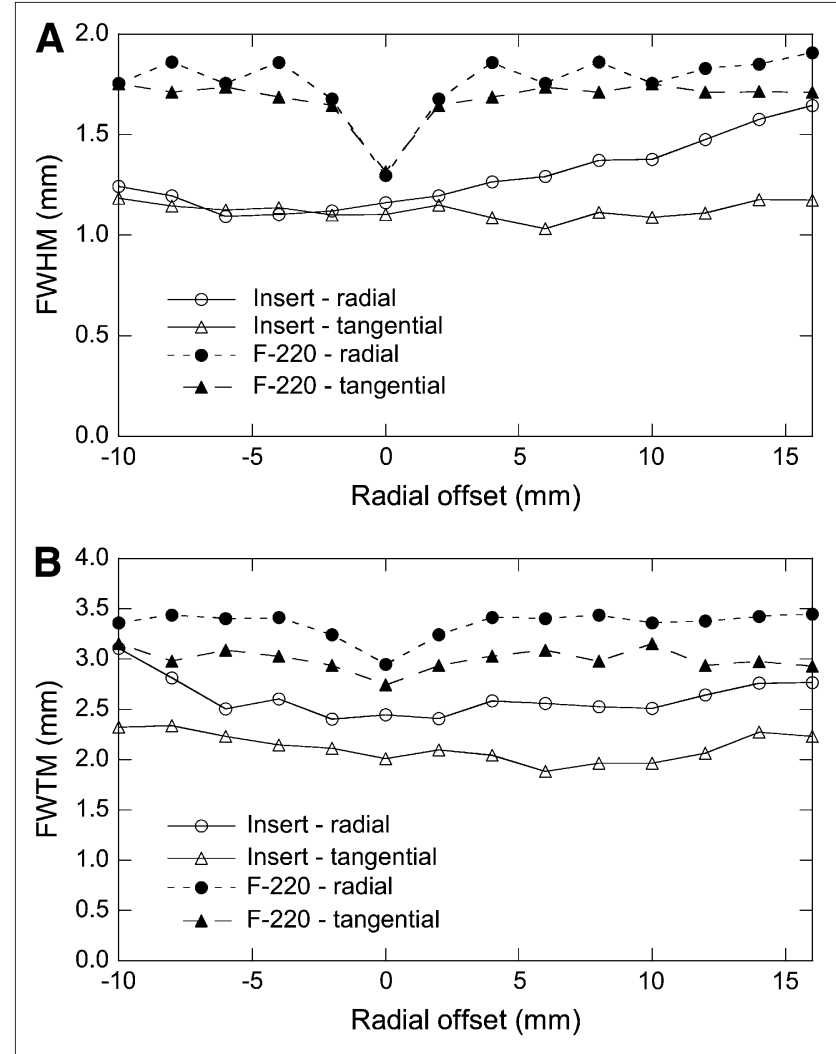

FIGURE 5. Resolution of PET insert device compared with F-220 scanner. Radial offset of point source was measured along the vertical direction with upper half of field of view having a positive offset. FWHM (A) and FWTM (B) were both measured from horizontal (tangential) and vertical (radial) profiles of pointsource images.

becomes 1.7-mm FWHM at 16-mm offset, approaching the radial resolution of the microPET F-220 system. In the lower half (negative radial offset), the radial resolution remains fairly uniform between 1.1- and 1.25-mm FWHM. The tangential resolution remains fairly uniform throughout the imaging field of view between $1.0-$ and $1.18-\mathrm{mm}$ FWHM. Figure 5B shows that the FWTM of the PET insert device is still asymmetric between the upper half and the lower half of the imaging field of view. However, the radial FWTM does not degrade as quickly as does the radial FWHM in the upper half of the field of view. Instead, it degrades more quickly in the lower half of the field of view when compared with the radial FWHM.

\section{Sensitivity of Insert System}

Figure 6 shows that the sensitivity of the PET insert system is approximately $0.064 \%$ at the center of the field of view and decreases to $0 \%$ at $16 \mathrm{~mm}$ from the center of the field of view in the axial direction. Therefore, the axial field of view is approximately $32-\mathrm{mm}$ wide along the central axis of the scanner even though the axial extent of the insert detector was only $20.5 \mathrm{~mm}$. This finding occurs because the coincidences were measured between the insert and 3 rings

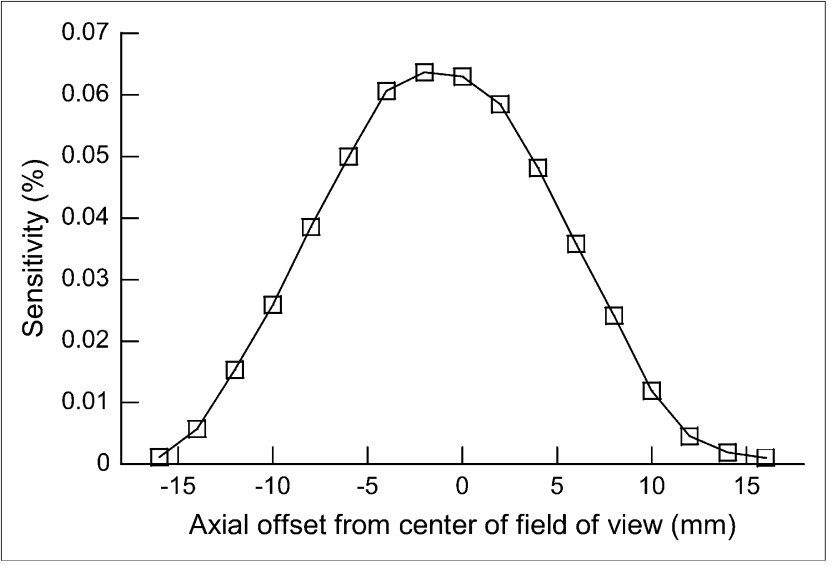

FIGURE 6. Sensitivity of PET insert device measured by a ${ }^{68} \mathrm{Ge}$ point source moving along central axis of field of view.

of detectors in the scanner that span an axial extent of 57 $\mathrm{mm}$. It should be noted, however, that this axial extent is reduced as the source is moved away from the central axis of the scanner. Therefore, this device cannot be used as if it had a true 32-mm axial field of view.

\section{Imaging Experiment}

Figure 7 shows 5 transverse slices through a mouse head, $3 \mathrm{~h}$ after ${ }^{18} \mathrm{~F}$-fluoride injection, measured by the detectors in the microPET F-220 scanner (Fig. 7A) and measured by the insert detector in coincidence with the detectors in the scanner (Fig. 7B). All images are displayed with the same gray scale. It is evident that the images from the insert device have higher contrast due to the improved image resolution that provides better-contrast recovery.

\section{DISCUSSION}

Although the length of LSO crystals in the insert detector was only $3.75 \mathrm{~mm}$, the parallax error became significant when the point source was positioned at a radial offset greater than $6 \mathrm{~mm}$ because the detector was only $29.6 \mathrm{~mm}$ away from the center of the field of view. This was the primary limitation precluding use of longer LSO crystals to improve the sensitivity of the insert system because the sensitivity and usable imaging field of view must be balanced for high image resolution. Therefore, the dilemma commonly seen in high-resolution PET scanner design - that is, trade-offs between high resolution and high sensitivitystill applies here.

Before integrating the high-resolution detector in the PET insert system, we performed an imaging experiment using this detector and a standard microPET Focus detector in our bench-top setup. The 2 detector arrays were mounted to 2 concentric rotation stages at the corresponding radii of the scanner ring and the PET insert device. The 2 detectors were rotated independently for $360^{\circ}$ to image a point source made of ${ }^{64} \mathrm{Cu}$ solution in a glass micropipette $(1-\mathrm{mm}$ outer diameter). The image resolution of this bench setup was 

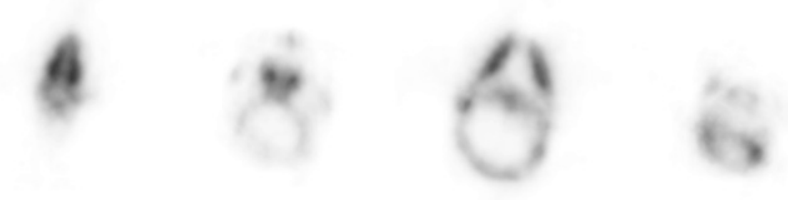

B

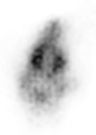

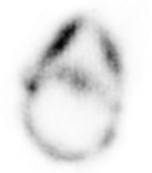

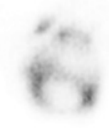

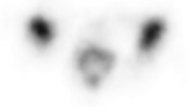

FIGURE 7. A 23.2-g mouse imaged $3 \mathrm{~h}$ after injection of $38.5 \mathrm{MBq}{ }^{18} \mathrm{~F}$-fluoride. (A) Images reconstructed by orderedsubset expectation maximization algorithm using coincidences recorded by detectors in microPET F-220 scanner. (B) Images reconstructed by maximum-likelihood expectation maximization algorithm using coincidences between PET insert detector and detectors in scanner. between 0.8- and 1.1-mm FWHM within the central 2-cm field of view, at least $20 \%$ higher than the image resolution measured from the PET insert system in the microPET F-220 scanner (data not shown). Three potential factors contributed to this degradation in image resolution between the bench setup and the actual prototype system. First, the point source used in the bench system was ${ }^{64} \mathrm{Cu}$ in a $1-\mathrm{mm}-$ diameter glass micropipette in air. The point source used in this study was a ${ }^{22} \mathrm{Na}$ point source $(0.5-\mathrm{mm}$ nominal diameter) embedded in an acrylic disk $(25 \mathrm{~mm}$ diameter $)$. The positron range of the ${ }^{64} \mathrm{Cu}$ in $1-\mathrm{mm}$ glass pipette surrounded by air may be smaller than the positron range of the ${ }^{22} \mathrm{Na}$ in a $25-\mathrm{mm}$ acrylic disk. Second, the digitizer in our bench setup was a 12-bit analog-to-digital-converter (ADC), whereas in the microPET F-220 scanner, an 8-bit flash ADC was used. This lower-resolution ADC in the scanner results in a poorer flood image and, hence, poorer crystal identification. A small fraction of events may be mislocated, which reduces the resolution of the system. Third, alignment of the PET insert device and the scanner is very critical for the image resolution. However, machining work has limited accuracy and, therefore, it is not possible to guarantee perfect alignment between the 2 systems. Our calibration procedure assumes that the centers of the 2 systems are perfectly aligned, however, and only calibrates for 1 degree of freedom (angular offset). Therefore, any misalignment between the centers of the 2 systems will lead to degradation of image resolution. A more sophisticated calibration procedure may be able to reduce this type of error but is beyond the scope of this work and will be explored in the future.

If we compare the peak sensitivity of this PET insert system $(0.064 \%)$ to that of the microPET F-220 scanner using the same energy and timing windows (3.0\%), the insert system is 46-fold lower than the original scanner. However, if we compare the sensitivity of this prototype PET insert device to animal SPECT systems, it is on the same order of magnitude as several state-of-the-art animal SPECT systems (19-21). If we increase the number of detectors in the PET insert system-for example, use 18 detectors in a ring - the sensitivity of the PET insert device (for insert-scanner coincidences) will increase fairly line- arly and reach $1.1 \%$. We have performed Monte Carlo simulation studies and showed that if we put a full-ring insert device in a PET scanner, 3 types of coincidence events can be registered (22): (a) between detectors in the scanner (scanner-scanner), (b) between detectors in the insert (insert-insert), and (c) between detectors of the insert and the scanner (insert-scanner). Each type of coincidence event permits estimation of radiotracer distribution in the object at a different resolution level. Ideally, one would like to use all coincidence events to best estimate the radiotracer distribution at the highest possible resolution. We have developed a maximum-likelihood expectation maximization reconstruction algorithm that jointly estimates the images using all 3 types of coincidence events (16). We expect that the sensitivity of a full-ring PET insert system will be similar to that of the original microPET F-220 scanner, whereas the image resolution of such a PET insert system should be significantly higher than that of the microPET F-220. The sensitivity of the entire system (insert plus scanner) can be further increased if we include 2 or more full rings of detectors in the insert system. This higher sensitivity is important to provide adequate statistics for the higher-resolution images. A full-ring insert device also provides complete sampling without rotation. Therefore, the system will provide dynamic imaging capability that is critical for biomedical research.

Because this prototype device can be attached only to the scanner after the transmission source holder is removed from the scanner, transmission scans become unavailable when using this insert device. One can, however, leave the mouse on the animal bed and perform the transmission scan after restoring the scanner to its original condition or perform calculated attenuation correction using coregistered CT images. Alternatively, if we design and mount a similar device on the translation stage that holds the animal bed, we will be able to preserve the transmission imaging capability of the scanner. However, the alignment of the 2 systems will become more challenging and is likely to require the more sophisticated calibration procedure describe here.

The correctness of biologic models derived from PET images relies completely on the quantitative accuracy of a 
PET system. A PET insert device inside the original field of view of a PET scanner inevitably complicates the quantitation of PET images because such a device can introduce additional attenuation and scatter of the $\gamma$-rays. Normalization of the coincidence detection efficiency between the 2 systems is also significantly more complicated than the original PET scanner. Therefore, it is a great challenge to ensure the quantitative accuracy of PET images from an insert device. The primary goal of this study was to prove the feasibility of improving image resolution of a scanner through the use of an insert device. The development of various correction techniques for PET insert devices is beyond the scope of this work. Because this prototype system has very low sensitivity and is not capable of dynamic imaging, it is unlikely that we will use it for routine animal studies. We will develop correction techniques and validate the quantitative accuracy of PET insert devices after we develop a full-ring or half-ring system that can be used for routine imaging experiments.

The use of an insert device inside a general-purpose PET scanner will decrease the original imaging field of view in exchange for higher resolution. For example, the microPET F-220 scanner has a transverse imaging field of view of 19 $\mathrm{cm}$, which is large enough to image small primates. The prototype insert system has a transverse imaging field of view of approximately $4 \mathrm{~cm}$, which is good only for imaging small rodents. The axial field of view is also reduced from the original $7.6-$ to $2.0-\mathrm{cm}$ for this particular system. Although the axial field of view of an insert system can be extended by using more detector blocks, the transverse field of view cannot be increased significantly due to the use of virtual-pinhole PET geometry. On the other hand, if one sees an insert system as an accessory of a PET scanner, this type of devices has the potential to increase the versatility of a general-purpose PET scanner-that is, a PET scanner with a large field of view can be used to perform whole-body imaging for large animals (or human subjects if it is a clinical scanner) - yet it still has the option to provide higher-resolution images within a smaller field of view by using a relatively low-cost insert device. Given the promising results in this feasibility study, we will explore the potential of PET insert devices in both preclinical and clinical PET applications by building more complete prototype insert systems that are capable of high-resolution dynamic imaging capability.

\section{CONCLUSION}

We have developed a prototype PET insert system to prove the feasibility of using an insert device in an existing small-animal PET scanner to provide higher-resolution PET capability. The resolution of the PET insert system ranges from 1.1- to 1.4-mm FWHM within the central 2-cm field of view. This system provides a significant improvement in image resolution based on point-source images and a mouse bone scan. The sensitivity of the prototype device is $0.064 \%$, significantly lower than a typical animal PET scanner due to the use of a single detector in the device. An insert system with more detector modules is expected to provide adequate sensitivity and improved image resolution for routine animal imaging studies. Carefully designed PET insert devices may have the potential to become an alternative approach for high-resolution PET instead of using special-purpose high-resolution PET systems.

\section{ACKNOWLEDGMENTS}

The authors thank Stefan Siegel and Danny F. Newport of Siemens Molecular Imaging, Inc., for their inspiring discussion; and Bill Margenau, Dennis Tapella, and John Kreitler for their technical support. This work was supported in part by the National Cancer Institute of NIH (grants R24-CA83060 and R33-CA110011) and by the Susan G. Komen for the Cure (grant BCTR0601279).

\section{REFERENCES}

1. Bloomfield PM, Rajeswaran S, Spinks TJ, et al. The design and physical characteristics of a small animal positron emission tomograph. Phys Med Biol. 1995;40:1105-1126.

2. Bruyndonckx P, Liu X, Tavernier S, Zhang S. Performance study of a 3D small animal PET scanner based on $\mathrm{BaF}_{2}$ crystals and a photo sensitive wire chamber. Nucl Instrum Methods [A]. 06 1997;392(1-3):407-413.

3. Lecomte R, Cadorette J, Rodrigue S, et al. Initial results from the Sherbrooke avalanche photodiode positron tomograph. IEEE Trans Nucl Sci. 1996;43:19521957.

4. Cherry SR, Shao Y, Silverman RW, et al. MicroPET: a high resolution PET scanner for imaging small animals. IEEE Trans Nucl Sci. 1997;44:11611166.

5. Watanabe M, Okada H, Shimizu K, et al. A high resolution animal PET scanner using compact PS-PMT detectors. IEEE Trans Nucl Sci. 1997;44:12771282.

6. Del Guerra A, Di Domenico G, Scandola M, Zavattini G. YAP-PET: first results of a small animal positron emission tomograph based on YAP:Ce finger crystals. IEEE Trans Nucl Sci. 1998;45:3105-3108.

7. Jeavons AP, Chandler RA, Dettmar CAR. A 3D HIDAC-PET camera with submillimetre resolution for imaging small animals. IEEE Trans Nucl Sci. 1999;46:468-473.

8. Tai YC, Chatziioannou A, Yang Y, et al. MicroPET II: design, development and initial performance of an improved microPET scanner for small-animal imaging. Phys Med Biol. 2003;48:1519-1537.

9. Rouze NC, Schmand M, Siegel S, Hutchins GD. Design of a small animal PET imaging system with 1 microliter volume resolution. IEEE Trans Nucl Sci. 2004;51:757-763.

10. Vaska P, Woody CL, Schlyer DJ, et al. RatCAP: miniaturized head-mounted PET for conscious rodent brain imaging. IEEE Trans Nucl Sci. 2004;51:27182722.

11. Miyaoka RS, Janes ML, Lee K, Park B, Kinahan PE, Lewellen TK. Development of a prototype micro crystal element scanner (MiCES): QuickPET II. Mol Imaging. 2005;4:117-127.

12. Phelps ME. PET: the merging of biology and imaging into molecular imaging. J Nucl Med. 2000;41:661-681.

13. Weissleder R, Mahmood U. Molecular imaging. Radiology. 2001;219:316333.

14. Tai Y-C, Wu H, Pal D, O'Sullivan JA. Virtual-pinhole positron emission tomography. $\mathrm{J} \mathrm{Nucl} \mathrm{Med}$. In press.

15. Tai YC, Ruangma A, Rowland D, et al. Performance evaluation of the microPET-Focus: a third generation microPET scanner dedicated to animal imaging. J Nucl Med. 2005;46:455-463.

16. Pal D, O'Sullivan JA, Wu H, Janecek M, Tai Y-C. 2D linear and iterative reconstruction algorithms for a PET-insert scanner. Phys Med Biol. 2007;52: 4293-4310. 
17. Daube-Witherspoon ME, Muehllehner G. Treatment of axial data in threedimensional PET. J Nucl Med. 1987;28:1717-1724.

18. Defrise M, Kinahan PE, Townsend DW, Michel C, Sibomana M, Newport DF. Exact and approximate rebinning algorithms for 3-D PET data. IEEE Trans Med Imaging. 1997;16:145-158.

19. Beekman FJ, van der Have F, Vastenhouw B, et al. U-SPECT-I: a novel system for submillimeter-resolution tomography with radiolabeled molecules in mice. J Nucl Med. 2005;46:1194-1200.
20. Funk T, Despres P, Barber WC, Shah KS, Hasegawa BH. A multipinhole small animal SPECT system with submillimeter spatial resolution. Med Phys. 2006;33:1259-1268.

21. Kim H, Furenlid LR, Crawford MJ, et al. SemiSPECT: a small-animal singlephoton emission computed tomography (SPECT) imager based on eight cadmium zinc telluride (CZT) detector arrays. Med Phys. 2006;33:465-474.

22. Janecek M, Wu H, Tai YC. A simulation study for the design of a prototype insert for whole-body PET scanners. IEEE Trans Nucl Sci. 2006;53:1143-1149. 